\title{
Polymorphism of Renalase Gene in Patients of Chronic Kidney Disease
}

\author{
Ravinder Singh Ahlawat ${ }^{1}$, Swapan Gupta ${ }^{2}$, Seema Kapoor ${ }^{3}$, Premashish Kar $^{2}$ \\ ${ }^{1}$ Department of Medicine, Nephrology Division, Maulana Azad Medical College, New Delhi, India \\ ${ }^{2}$ Department of Medicine, Maulana Azad Medical College, New Delhi, India \\ ${ }^{3}$ Department of Pediatrics and Molecular Genetics, Maulana Azad Medical College, New Delhi, India \\ Email: ahlawat.ravi@gmail.com
}

Received July 24, 2012; revised September 4, 2012; accepted September 14, 2012

\begin{abstract}
Introduction: Chronic kidney disease (CKD) is an important public health problem. Early detection and treatment is a key factor for prevention of its complications. Hypertensive nephrosclerosis is a subtype of CKD which has a poor correlation between hypertension and development of nephropathy, implying role of genetic factors or epigenetic factors. The knowledge regarding genetic factors is limited. Renalase is a novel hormone with its gene on chromosome 10 , which secretes flavin adenine dinucleotide dependent amine oxidase. Renalase metabolizes circulating catecholamines and modulates blood pressure and cardiac function. Recently, two single nucleotide polymorphisms of renalase gene rs2576178 GG and rs2296545 CC have been linked to essential hypertension. The SNPrs2296545 CC is also shown to be associated with cardiac hypertrophy, dysfunction and ischemia. The association of these two single nucleotide polymorphisms with hypertensive nephrosclerosis has not been investigated. Methods: We designed a case-control study to investigate whether the two known renalase gene polymorphisms rs2576178 and rs2296545 are associated with CKD particularly hypertensive nephrosclerosis. We genotyped these two polymorphisms in 287 subjects from North Indian population (106 CKD cases and 181 controls). Results: Comparison shows that subjects with hypertensive nephrosclerosis had higher frequencies of rs $2296545 \mathrm{C}$ allele than the healthy controls $(0.63$ versus $0.47, \mathrm{p}<0.02)$. The odds ratio for rs $2296545 \mathrm{CC}$ genotype in hypertensive nephrosclerosis were 2.55 (95\% CI, 1.03 to $6.42 ; \mathrm{p}=0.02)$ (CC versus GG) and $2.11(95 \% \mathrm{CI}, 1.01$ to $4.42 ; \mathrm{p}=0.03)(\mathrm{CC}$ versus $\mathrm{CG}+\mathrm{GG})$ compared to controls. Conclusion: These findings may provide novel insight into the role of additional genomic regions as susceptibility gene in the pathophysiology of hypertensive nephrosclerosis. Further, to account for geoethnic variation, studies on heterogeneous populations involving a larger sample size are required. The correlation between this structural change and actual levels of the enzyme or the activity are required to strengthen this association as well as to be clinically applicable.
\end{abstract}

Keywords: Chronic Kidney Disease (CKD); Hypertension; Hypertensive Nephrosclerosis; Renalase; Single Nucleotide Polymorphisms (SNP); End Stage Renal Disease (ESRD)

\section{Introduction}

Chronic kidney disease (CKD) is a multifactorial disorder affected by various environmental and genetic factors. A genetic predisposition for renal failure is demonstrated by the 3 - 9 times higher probability of ESRD in patients with a family history of CKD, compared to the general population [1-4]. However, it is difficult to assess whether this predisposition is due to a specific genetic susceptibility to renal damage, or to other comorbid conditions generally accepted to have poly- or oligo-genetic components, like hypertension, diabetes or atherosclerosis. Still, this observation has launched the search for nephropathy susceptibility genes.

Prevalence of CKD increases in ageing population and approximately $38 \%$ of adults aged 70 years and older have an eGFR less than $60 \mathrm{ml} / \mathrm{min} / 1.73 \mathrm{~m}^{2}$. A number of factors like renovascular disease, hypertension, recurrent urinary tract infection and obstructive uropathy are implicated for CKD in ageing population $[5,6]$.

Hypertension is both a cause and consequence of CKD. Hypertension is well recognized as a risk factor for development and progression of CKD [7]. With advancing chronic kidney disease, hypertension is a unique feature and various mechanisms like activation of renin angiotensin system, expansion of extracellular volume and increased sympathetic activity operate for its development.

The term hypertensive nephrosclerosis is usually used for kidney disease which complicates arterial hypertension (AHT) and primarily affects the preglomerular microvasculature $[8,9]$. Its causal relation with essential hyper- 
tension is still a subject of debate, as still it is not clear that how a well-treated AHT can lead to end-stage CKD [10-13]. Renal function can remain stable for years if hypertension is well controlled. However, in a few cases the disease progresses to end-stage CKD $[14,15]$. The lack of correlation between the degree of hypertension control and the prevention of disease progression, from the clinical and histological standpoint suggests that this process may be an intrinsic and early renal disease $[9,16]$. For many years, genetic markers have been sought that could explain the onset and progression of the disease.

A study by Freedman and colleagues reported that first and second degree family members of patients with CKD are 2 - 3 times as likely to have incident ESRD and have high rate of impaired kidney function suggesting inherent genetic factors in initiation, susceptibility and progression of kidney disease irrespective of underlying cause of CKD [17]. Another study by Kao et al. [18] revealed a close relationship between CKD and hypertensive nephrosclerosis in non-diabetic subject and polymorphism of MYH9 gene located on chromosome 22 was observed. In India such renal specific gene polymorphism study in CKD patients are scanty [19].

Renalase is one of the recently discovered enzyme causally related to kidney function [20]. It is a novel flavin adenine dinucleotide (FAD) dependent amine oxidase with its gene having 9 exons spanning approximately 311,000 base pairs and resides on chromosomes 10 at q23.33. The expression of this gene is highest in the kidneys and once secreted by the kidney, it circulates in the blood and affects systolic BP and cardiac function by modulating catecholamines [21]. Recombinant renalase has been shown to lower blood pressure and heart rate and improve the cardiovascular function [22-24]. Low renalase levels are associated with hypertension and increased cardiovascular morbidity [25-27].

Renalase levels are reported to be low in CKD patients implicating its role in development of hypertension and associated morbidity and mortality in such patients [20, 25]. Single nucleotide polymorphisms (SNPs) of renalase gene have been described recently. SNPs rs2576178 GG and rs2296545 CC genotypes have been found to be associated with essential hypertension in Northern Han Chinese population [28]. Also rs2296545 CC was found to be associated with cardiac hypertrophy, dysfunction and ischemia in Caucasians by Farzaneh-Far et al. [29]. The polymorphism is shown to result in abnormal gene expression affecting enzymatic activity of renalase. Low renalase expression correlating with increased plasma norepinephrine, systolic blood pressure and proteinuria suggests that renalase plays an important role in catecholamine metabolism and is involved in development of hypertension and decreased cardiovascular function and may directly or indirectly contributes to renal injury [27]. These findings raise the intriguing possibility that decreased activity of the enzyme renalase may be due to abnormalities in renalase expression and this may increase the risk of development or progression of kidney disease.

We studied whether the polymorphism in renalase gene is associated with CKD in general or specifically with hypertensive nephropathy or nephrosclerosis, possibly due to following reasons:

1) Hypertension is common in patients with chronic renal disease. But there is lack of direct correlation between the degree of hypertension, the control and the prevention of disease progression emphasizing the role of genetic factors;

2) CKD initiation especially hypertensive nephrosclerosis cannot be accounted for by the present known genetic and environmental factors;

3) Renalase gene is primarily involved in regulation of hypertension and its polymorphism has been shown to be associated with hypertension. In other words, we intended to study the association of renalase gene polymorphisms with chronic kidney disease and hypertensive nephrosclerosis in particular.

\section{Materials and Methods}

\subsection{Sample Size}

A total of 300 subjects were enrolled for the study. It was a case control study approved by the Research Ethic Committee of Maulana Azad Medical College.

\subsubsection{Cases}

Cases were selected according to the following criteria (Table 1).

Chronic kidney disease was diagnosed using K/DOQI (Kidney Disease Outcomes Quality Initiative) clinical practice guidelines for CKD [31]. Cases of CKD were divided into two groups:

1) Cases with hypertensive nephrosclerosis;

2) Cases with other causes of CKD.

Hypertensive nephrosclerosis was diagnosed according to modified clinical criteria by AASK task group [32], given as follows (Table 2).

\subsubsection{Controls}

Subjects with hypertension but without any evidence of kidney disease and normal healthy adults with same geoethnic groups were taken as controls.

The Study was conducted on patients in a tertiary care center in northern India. The patients included in the study belonged to northern Indian ethnicity. Informed consent was taken from each participant.

BP was measured using a mercury sphygmomanometer after 5 minutes of rest in a seated position in right arm, using an adequate cuff by the trained observer. 
Table 1. Criteria for selection of cases.

\begin{tabular}{cc}
\hline Inclusion criteria & Exclusion criteria \\
\hline Adult patients (age group 18 - & $\geq 65$ years old to minimize the \\
effect of age related gene \\
Denaturation [30]
\end{tabular}

Table 2. Criteria for diagnosis of hypertensive nephrosclerosis.

\begin{tabular}{|c|c|}
\hline Inclusion criteria & Exclusion criteria \\
\hline $\begin{array}{l}\mathrm{AHT} \geq 140 / 90 \mathrm{~mm} \mathrm{Hg} \text { or on } \\
\text { antihypertensive medication }\end{array}$ & $\begin{array}{c}\text { AHT }<140 / 90 \mathrm{~mm} \mathrm{Hg} \text { without } \\
\text { treatment }\end{array}$ \\
\hline $\begin{array}{l}\text { Urine protein to urine } \\
\text { creatinine ratio }<2.0\end{array}$ & $\begin{array}{l}\text { Urine protein to urine creatinine } \\
\text { ratio } \geq 2.0 \text {, except in cases in } \\
\text { which there is histological } \\
\text { confirmation by kidney biopsy }\end{array}$ \\
\hline Age 18 - 65 years & Age $\geq 65$ years \\
\hline $\begin{array}{l}\text { CKD (according to K\DOQI } \\
\text { criteria) }\end{array}$ & $\begin{array}{l}\text { Evidence of other identifiable } \\
\text { causes of chronic kidney disease } \\
\text { like diabetes, immune complex } \\
\text { disease, solitary kidney }\end{array}$ \\
\hline
\end{tabular}

Arterial hypertension was defined as SBP $\geq 140 \mathrm{~mm}$ $\mathrm{Hg}$ and/or DBP $\geq 90 \mathrm{~mm} \mathrm{Hg}$, or treatment with diet or antihypertensive agents.

Diabetes mellitus was defined as fasting glucose $\geq 126$ $\mathrm{mg} / \mathrm{dl}$ or $\geq 200 \mathrm{mg} / \mathrm{dl} 2$ hours after oral glucose or already on anti diabetic treatment (diet, oral anti diabetic agents or insulin).

Smoker was identified to be an individual who have been smoking tobacco in any form like bidis, hookah, cigarettes, cigars, pipe during the last six months. Other tests like hemoglobin, blood sugar, serum creatinine, serum protein, blood cholesterol and triglycerides were estimated with standard biochemical lab techniques.

\subsection{Methodology of the Determination of the Genotype}

Blood samples for genetic studies were collected and submitted to the Genetics Laboratory, Lok Nayak Hospital. DNA was obtained from leukocytes from EDTA anticoagulated peripheral blood. Standard methods of genomic DNA extraction, PCR and RFLP were employed. The DNA was extracted with an equal amount of a phenol/chloroform/isoamyl (25:24:1) alcohol mixture to remove contaminating proteins. The mixture was then vortexed vigourously for 30 seconds and centrifuged for 5 minutes at a $12,000 \times \mathrm{g}$. The top (aqueous) phase was removed and transferred into a new tube and was extracted again with an equal volume of chloroform. After extraction, the top phase was removed and transferred into a new test tube for ethyl alcohol precipitation. In order to precipitate nucleic acid from an aqueous solution, it had to be adjusted to a $0.2 \mathrm{M}$ salt concentration with
$3 \mathrm{M}$ sodium acetate ( $\mathrm{pH} 4.8$ ). Then 2.5 vol. of $100 \%$ ethyl alcohol was added and nucleic acids were precipitated for half hour at $-20^{\circ} \mathrm{C}$. Afterwards the sample was centrifuged for 10 minutes at a speed of $10,000 \times \mathrm{g}$. To remove excessive salt, the pellet was washed with $70 \%$ ethanol. Finally, the pellet was air dried at room temperature or in speed vacuum. Nucleic acids were stored in water or in Tris buffer at $-20^{\circ} \mathrm{C}$. DNA in aqueous solution was precipitated with an equal volume of isopropanol. The mixture was precipitated on ice for 20 minutes and centrifuged at $10,000 \times \mathrm{g}$ for 10 minutes. The DNA pellet was washed with $70 \%$ ethanol to remove salts, air dried and resuspended in an appropriate volume of water or Tris buffer. The estimation of concentration of genomic DNA in solution, obtained from different sources was done using a dual ultra-violet spectrophotometer (Cecil, USA) at wavelength $260 \mathrm{~nm}$ and $280 \mathrm{~nm}$ and concentration of DNA was calculated using the Formula (1):

$$
\begin{aligned}
& \text { Absorbance at } 260 \mathrm{~nm} \times \text { Dilution factor } \times 50 \\
& =\operatorname{DNA}(\mu \mathrm{g} / \mathrm{ml})
\end{aligned}
$$

Alternatively an approximate concentration of genomic DNA was determined by electrophoresis of the 1 microliter or 2 microliter of DNA solution on bromide - stained $1 \%$ agarose gel in $1 \mathrm{X}$ Tris buffer with $1 \mu \mathrm{g}$ Hind III digested $\lambda$-DNA molecular weight marker as reference. PCR RFLP was employed to look for SNPs. Probes designed against 2 previously identified SNPs (rs 2576178 $\&$ rs 2296545) were used. The PCR conditions were $95^{\circ} \mathrm{C}$ for 0.50 minutes, $60^{\circ} \mathrm{C}$ for 0.50 minutes, $72^{\circ} \mathrm{C}$ for 3.00 minutes and final extension of $72^{\circ} \mathrm{C}$ for $5.00 \mathrm{~min}$ utes.

\subsection{Statistical Analysis}

There was a descriptive analysis of continuous variables, giving the mean and standard deviation, and in case of discrete variables, the frequency distribution and percentages were given. When necessary, $95 \%$ confidence intervals were calculated. The association between qualitative variables was assessed with the chi-square or Fisher's exact test and quantitative variables using parametric tests (t-test and ANOVA). The genotype distribution and allele frequency among study population groups were determined and compared by the Chi square test. Level of significance was accepted at $95 \%$ C.I. with $\mathrm{p}<0.05$. For statistical analysis, SPSS for Windows, version 19.0 was used.

\section{Observation and Results}

A total of 300 subjects were enrolled initially, but later on 13 patients were excluded from the study as the DNA failed to amplify in these cases. The total number of 
study population was limited to 287 patients. The study population was categorized into groups for comparison and analysis.

The subjects were distributed as follows:

1) Group 1: cases (subjects with chronic kidney disease), 106 cases

Group 1 was further subdivided into:

a) subjects with $\mathrm{CKD}$ and hypertensive nephrosclerosis 59 cases

b) subjects with CKD other than hypertensive nephrosclerosis 47 cases

2) Group2: hypertensive controls with no kidney disease 91 subjects and

3) Group3: general population subjects 90 (healthy controls).

\subsection{Baseline Clinical Characteristics of Subjects}

Group 1 included 106 subjects with mean age $47.8 \pm 13.1$, $58(54.7 \%)$ men, $26(24.5 \%)$ diabetic and 28 (26.4\%) smokers. The subgroup 1A included 59 subjects with mean age $47.4 \pm 13.4,30(50.8 \%)$ men, and15 (25.4\%) smokers. The subgroup 1B included 47 subjects with mean age $51.2 \pm 8.3,33(36.3 \%)$ men, 26 (55.3\%) diabetic and $13(27.7 \%)$ smokers. Group 2 included 91 subjects with mean age $51.2 \pm 8.3,33(36.3 \%)$ men and 23 (25.3\%) smokers. Group 3 included 90 subjects with mean age $39.8 \pm 14.1,38(42.2 \%)$ men and $21(23.3 \%)$ smokers. There was statistically significant difference among different study groups and various laboratory parameters studied i.e. mean haemoglobin, creatinine clearance, serum proteins, serum cholesterol and serum triglyceride levels. History of current smoking was not statistically significant among the groups $(p>0.05)$. Baseline clinical characteristics of subjects who entered into the final analysis are presented in Table 3.

\subsection{Polymorphisms of Renalase Gene Examined in the Study}

We genotyped two previously identified rs2576178 and rs2296545 single nucleotide polymorphisms of renalase in 285 and 287 subjects respectively ( 2 out of 287 subjects studied showed ambiguous results with rs2576178 polymorphism and were excluded). Different study population groups were categorized by genotype and allele frequency at rs2576178 and rs2296545 locus.

\subsubsection{Observations for rs2576178 Genotype}

Results of different study population groups were categorized by genotype and allele frequency at rs2576178 locus and compared (Tables 4 and 5). Comparison shows no significant difference among various study population groups for genotype distributions and allele frequencies at SNP rs2576178, suggesting that there is no significant association of SNP rs2576178 locus and hypertension, CKD, and hypertensive nephrosclerosis

\subsubsection{Observations for rs2296545 Genotype}

Different study population groups were categorized by genotype and allele frequency at rs2296545 locus and compared (Tables 6 and 7). Based on the observations, significant difference was found between Group 1A (subjects with hypertensive nephrosclerosis) and Group 3 (Healthy controls) for the frequency of C allele $(\mathrm{p}<0.02)$. The frequency of rs2296545 C allele was higher in group 1(a). The genotype distribution of rs2296545 between these two groups also showed a trend towards statistical difference $(p=0.07)$.

Table 3. Baseline clinical characteristics of study population.

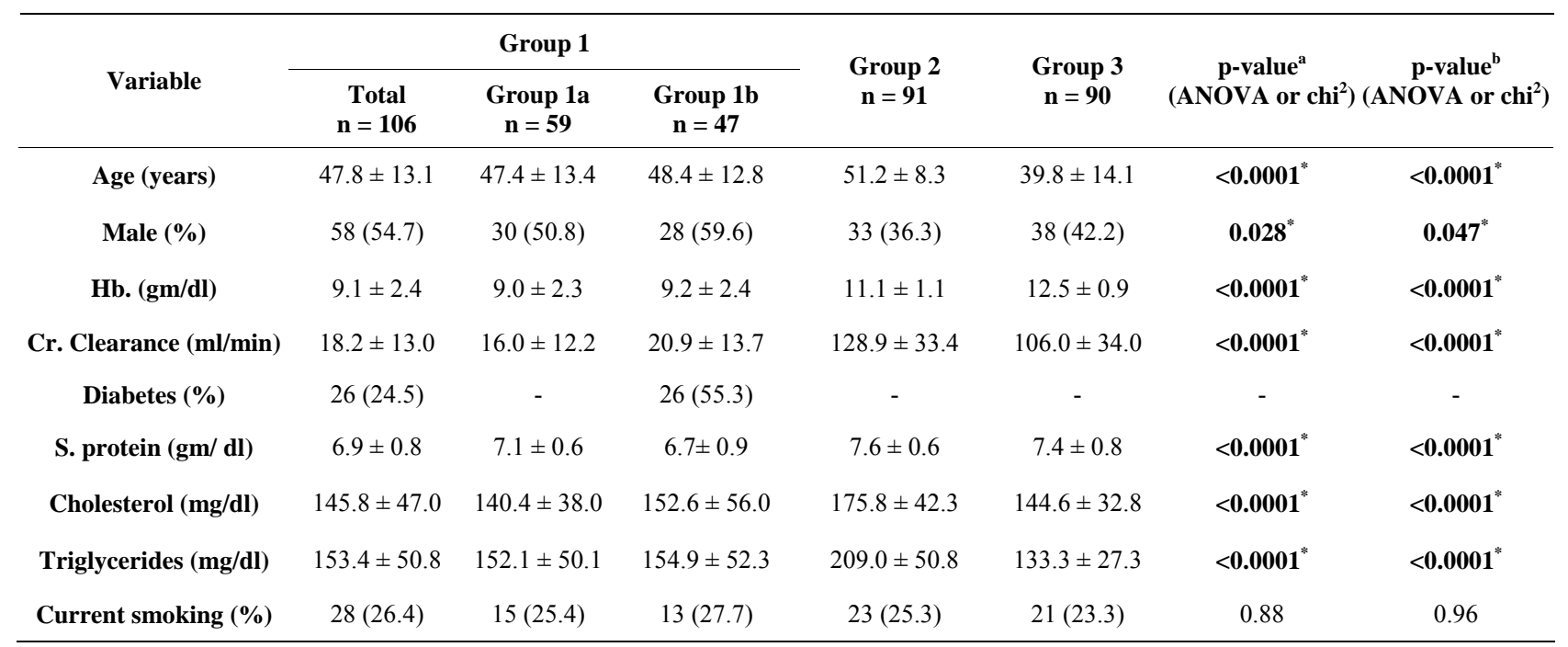

${ }^{*}$ p value statistically significant; ${ }^{\mathrm{a}}=$ signifies $\mathrm{p}$ value among group 1 (total), 2 and $3 ;^{\mathrm{b}}=$ signifies $\mathrm{p}$ value among group $1 \mathrm{a}, 1 \mathrm{~b}, 2$ and 3 . 
Table 4. Study population groups and their genotype distributions and allele frequencies at rs2576178 locus.

\begin{tabular}{|c|c|c|c|c|c|}
\hline & \multicolumn{3}{|c|}{ Genotype } & \multicolumn{2}{|c|}{ Allele frequency ${ }^{*}$} \\
\hline & GG & GA & AA & G & A \\
\hline $\begin{array}{c}\text { Group 1 } \\
\mathrm{n}=106(\%)\end{array}$ & $34(32.08)$ & $38(35.85)$ & $34(32.08)$ & 0.50 & 0.50 \\
\hline $\begin{array}{c}\text { Group 1a } \\
\mathrm{n}=59(\%)\end{array}$ & $24(40.68)$ & $20(33.90)$ & $15(25.42)$ & 0.58 & 0.42 \\
\hline $\begin{array}{c}\text { Group 1b } \\
\mathrm{n}=47(\%)\end{array}$ & $10(21.28)$ & $18(38.30)$ & $19(40.43)$ & 0.40 & 0.60 \\
\hline $\begin{array}{c}\text { Group } 2 \\
\mathrm{n}=\mathbf{8 9}(\%)\end{array}$ & $23(25.84)$ & $38(42.70)$ & $28(31.46)$ & 0.47 & 0.53 \\
\hline $\begin{array}{c}\text { Group } 3 \\
\mathbf{n}=90(\%)\end{array}$ & $25(27.78)$ & 35 (38.89) & $30(33.33)$ & 0.47 & 0.53 \\
\hline
\end{tabular}

Table 5. Comparison of genotype distributions and allele frequencies at rs2576178 locus among study population groups.

\begin{tabular}{ccc}
\hline Comparison for statistical test & $\begin{array}{c}\text { p-value } \\
\text { (Genotype) }\end{array}$ & $\begin{array}{c}\text { p-value } \\
\text { (Allele) }\end{array}$ \\
\hline Group 1 versus Group 2 & 0.54 & 0.67 \\
Group 1 versus Group 3 & 0.80 & 0.67 \\
Group 1a versus Group 2 & 0.17 & 0.12 \\
Group 1a versus Group 3 & 0.24 & 0.11 \\
Group 2 versus Group 3 & 0.87 & 1.0 \\
\hline
\end{tabular}

Table 6. Study population groups and their genotype distributions and allele frequencies at rs2296545 locus.

\begin{tabular}{|c|c|c|c|c|c|}
\hline & \multicolumn{3}{|c|}{ Genotype } & \multicolumn{2}{|c|}{ Allele frequency ${ }^{*}$} \\
\hline & CC & CG & GG & C & G \\
\hline $\begin{array}{c}\text { Group1 } \\
\mathrm{N}=106(\%)\end{array}$ & $40(37.74)$ & $34(32.08)$ & $32(30.19)$ & 0.54 & 0.46 \\
\hline $\begin{array}{c}\text { Group 1a } \\
\mathrm{N}=59(\%)\end{array}$ & $28(47.46)$ & $18(30.51)$ & $13(22.03)$ & 0.63 & 0.37 \\
\hline $\begin{array}{c}\text { Group 1b } \\
N=47(\%)\end{array}$ & $12(25.53)$ & $16(34.04)$ & $19(40.43)$ & 0.43 & 0.57 \\
\hline $\begin{array}{c}\text { Group } 2 \\
\mathrm{~N}=91(\%)\end{array}$ & $27(29.67)$ & $40(43.96)$ & $24(26.37)$ & 0.52 & 0.48 \\
\hline $\begin{array}{c}\text { Group3 } \\
\mathbf{N}=\mathbf{9 0}(\%)\end{array}$ & $27(30.00)$ & $31(34.44)$ & $32(35.56)$ & 0.47 & 0.53 \\
\hline
\end{tabular}

Further, Odds Ratio for hypertensive nephrosclerosis associated with rs2296545 CC genotype were $2.55(95 \%$ $\mathrm{CI}, 1.03-6.42 ; \mathrm{p}=0.02)(\mathrm{CC}$ versus $\mathrm{GG})$ and $2.11(95 \%$ $\mathrm{CI}, 1.01-4.42 ; \mathrm{p}=0.03$ ) (CC versus CG or GG) compared to healthy controls, suggesting that there is a significant association between presence of SNP rs2296545 CC genotype and hypertensive nephrosclerosis (Table 8, Figure 1). No significant difference was found between
Table 7. Comparison of genotype distributions and allele frequencies at rs2296545 locus among study population groups.

\begin{tabular}{ccc}
\hline Comparison for statistical test & $\begin{array}{c}\text { p-value } \\
\text { (Genotype) }\end{array}$ & $\begin{array}{c}\text { p-value } \\
\text { (Allele) }\end{array}$ \\
\hline Group 1 versus Group 2 & 0.22 & 0.70 \\
Group 1 versus Group 3 & 0.39 & 0.32 \\
Group 1a versus Group 2 & 0.08 & 0.12 \\
Group 1a versus Group 3 & 0.07 & $\mathbf{0 . 0 2}$ \\
Group 2 versus Group 3 & 0.32 & 0.47 \\
${ }^{*}$ p value statistically significant. & &
\end{tabular}

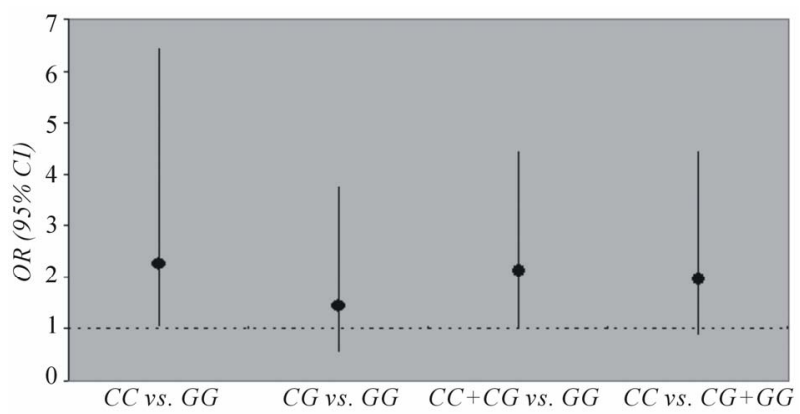

Figure 1. Odd ratios (ORs) for Group 1A (hypertensive nephrosc lerosis) with genotypes of SNP rs2296545. CI indicates confidence interval. The $95 \% \mathrm{CI}$ lines crossing the OR line of indicate no significance.

SNP rs2296545 genotypes and hypertension or CKD group as a whole.

\section{Discussion}

The causal relation of hypertensive nephrosclerosis with essential hypertension has been a subject of debate as the progression of kidney failure is slow in most cases of essential hypertension. Few genes have been implicated in association with development of hypertensive nephrosclerosis but still cannot account for the variability in all cases. Freedman et al. [33] clearly demonstrated role of genetic polymorphism like MYH9 gene polymorphism in development of hypertensive nephrosclerosis. But they also concluded that all MYH9 risk homozygotes do not develop kidney disease, environmental responses and/or interactions with other genes are clearly necessary to initiate MYH9 associated nephropathy in genetically susceptible host.

The present study found an association of renalase gene single nucleotide polymorphism rs2296545 CC genotype and hypertensive nephrosclerosis. It is a step further to define the susceptibility genetic loci for this disease. The mechanism behind this association is based on the principle that hypertensive nephrosclerosis belong to a category of vascular diseases. Renalase gene has been shown 
Table 8. Association of CC (vs. GG and CG or GG) genotype at rs2296545 with Group 1a (hypertensive nephrosclerosis) compared to Group 3 (healthy controls).

\begin{tabular}{|c|c|c|c|c|c|c|}
\hline \multirow{2}{*}{ Group } & \multicolumn{2}{|c|}{ Genotype } & \multirow{2}{*}{ p-value } & \multicolumn{2}{|c|}{ Genotype } & \multirow{2}{*}{ p-value } \\
\hline & $\mathrm{CC}$ & GG & & $\mathrm{CC}$ & CG or GG & \\
\hline 1a & 28 & 13 & $0.02^{*}$ & 28 & 31 & $0.03^{*}$ \\
\hline 3 & 27 & 32 & O.R. $2.25(1.03-6.42)$ & 27 & 63 & O.R. $2.11(1.01-4.42)$ \\
\hline
\end{tabular}

${ }^{*}$ p value statistically significant.

to play an important role in regulating sympathetic nervous system (SNS) activity, hypertension and cardiovascular function. This supports a mechanistic link between the renalase-catecholamine axis and vascular diseases. Abnormalities/functional changes in its activity due to the polymorphisms are thus expected to induce a cascade of vascular damages owing to unregulated SNS activity and could be one of the factors for various kinds of vascular complications. Hence, we had hypothesize that polymorphisms in these common genetic loci might affect the development of cardiovascular diseases (CVD) as well as chronic kidney disease especially hypertensive nephrosclerosis. Association with hypertension and increased cardiovascular morbidity has already been shown in previous studies and our study provides important insight into development of hypertensive nephrosclerosis in association with this genotype, suggesting that rs2296 545 CC genotype might be used as a common risk allele for CVD and hypertensive nephrosclerosis. Further, it may be hypothesized that the renalase activity is mostly regulated by its genotypes and the SNP rs2296545 CC genotype and its accompanying amino acids substitution is related to its enzymatic activity (low in $\mathrm{C} / \mathrm{C}$ genotype and high in $\mathrm{G} / \mathrm{G}$ genotype). Thus, theoretically, SNP rs2296545 CC genotype has a potentially lower SNS inhibitory effect and less vasoprotecting property. The recent evidence by Farzaneh-Far et al. [29] that rs2296 545 CC genotype is related to lower renalase activity, higher SNS activity and poor CVD prognosis also supports our hypothesis. In accordance with this association, our study postulate that rs2296545 CC genotype with a low renalase activity is a risk factor for renal insufficiency as well. We did not find the association of SNP rs2296545 with CKD other than hypertensive nephrosclerosis possibly because other etiologic and genetic factors may be responsible in these cases; this being of multifactorial etiology. Also, no association was found between SNP rs2576178 genotypes with either CKD or hypertensive nephrosclerosis, possibly because it is not a known functional polymerphism or due to inherent geoethnic differences.

No significant association of these two polymorphisms with hypertension was found in our study consistent with the findings of study by Farzaneh-Far et al. [29]. This may be attributed to different genetic make up and ethnic background of these populations and require future large studies to resolve the issue.

Our study implies that there is a complex and intricate relationship between renalase gene polymorphism

(rs2296545 CC genotype), hypertension and hypertensive nephrosclerosis. It is possible that polymorphism in renalase gene may directly cause vascular damage to kidneys even in the absence of hypertension, although this remains to be proven. Several studies revealed that high BP does not commonly lead to progressive nephropathy $[10,12,14]$. The few hypertensive patients who demonstrate progressive kidney disease are typically labeled as having hypertensive nephrosclerosis which is presumed to progress to hypertensive ESRD. Our association results suggest that patients labeled as hypertensive ESRD may have intricate deficits due to renalase gene polymorphisms predisposing them to the development of nephrosclerosis. Thus, non specific and incompletely characterized causes of CKD, typically attributed to hypertension and chronic glomerular diseases, may be accounted for by renalase gene polymorphism along with other genetic associations previously described. Evaluation of renal histology in those with early stages of polymerphism associated nephropathy will clarify this issue.

The clinical implication of our study is that renalase gene polymorphism (rs2296545 CC genotype) might increase the susceptibility of an individual to develop hypertensive nephrosclerosis. But, once it is identified, there is a need to identify clinical parameters and biomarkers (e.g., renalase levels and its enzymatic activity) that may help a clinician in predicting the early development and progression of nephropathy before it is manifest in the form of deranged renal functions or proteinuria. Future studies will be required to answer this question.

One limitation of our study was that the sample size was small and was from a hospital based setting. Genetic studies require large population based study designs with no genetic drift, random mating, reproductive isolation from other populations i.e. no gene flow or migration and no differential survival or reproduction among phenoltypes i.e. no natural selection. All these are the assumptions that populations must meet for the Hardy Weinberg equilibrium, a law in genetics. It is very difficult to control all these factors in a heterogeneous population of 
North Indian region. But prior selection of known polymorphism in renalase makes the genotype-phenotype association biologically plausible and false positives unlikely.

Another limitation is the potential for recruitment bias. First, the study population was restricted to Indian race; therefore our results may not be generalized to other racial groups. Differences in age and gender distributions might affect the results also. Individuals classified as having hypertensive CKD in the absence of quantified proteinuria may actually have had proteinuria and underlying focal segmental glomerulosclerosis.

We did not measure the renalase levels or its enzymatic activity. Correlation of genotypes with absolute renalase levels or its enzymatic activity would have further strengthened the study.

There are many remaining questions in this rapidly evolving hypothesis. Most importantly, should patients of newly diagnosed hypertensive nephrosclerosis undergo testing for renalase gene polymorphism? Who are the candidates who should undergo polymorphisms testing before development of nephropathy? Are there any clinical parameters and biomarkers that may predict the early development and progression of nephropathy once an individual is found to have the risk genotype? Should those found to be homozygous for risk allele be treated before development of nephropathy? Are patients with renalase gene associated renal disease responsive to recombinant renalase replacement therapy? Prospective studies and clinical trials will be required to answer these questions.

Thus, renalase is a gene with pronounced effect in common and complex kidney disease. The causative SNP and mechanism of action of renalase gene associated kidney disease has not yet been fully elucidated, although new and exciting era has dawned with this important discovery. The early identification and intervenetion may substantially reduce burden of disease in this population.

\section{Conclusions}

In conclusion, we found the association of the renalase single nucleotide polymorphism (SNP) rs2296545 (C allele and CC genotype) with hypertensive nephrosclerosis in a case control study in the North Indian population. However, no association was found between other SNP rs 2576178 and CKD. The present study suggests that genetic variations in the renalase gene may influence the susceptibility to hypertensive nephrosclerosis. These findings will potentially contribute to a better understanding of the pathophysiology of hypertensive nephrosclerosis and may aid in early identification of the disease process.

In addition, to attribute for the geoethnic variations, a larger sample size is required to confirm and interpret the association in other populations. Determination of rena- lase hormone as a biomarker, elucidation of other biomarkers predicting development of nephropathy in individuals with risk genotype and the potential therapeutic role for renalase replacement in such persons may prove to be an asset for the practicing clinician.

\section{REFERENCES}

[1] S. G. Satko, J. R. Sedor, S. K. Iyengar and B. I. Freedman, "Familial Clustering of Chronic Kidney Disease," Seminars in Dialysis, Vol. 20, No. 4, 2007, pp. 229-236. doi:10.1111/j.1525-139X.2007.00282.X

[2] C. S. Fox, Q. Yang, L. A. Cupples, C. Y. Guo, M. G. Larson, E. P. Leip, et al., "Genome Wide Linkage Analysis to Serum Creatinine, GFR, and Creatinine Clearance in a Community-Based Population: The Framingham Heart Study," Journal of the American Society of Nephrology, Vol. 15, No. 9, 2004, pp. 2457-2461. doi:10.1097/01.ASN.0000135972.13396.6F

[3] P. P. Faronato, M. Maioli, G. Tonolo, E. Brocco, F. Noventa, F. Piarulli, et al., "Clustering of Albumin Excretion Rate Abnormalities in Caucasian Patients with NIDDM. The Italian NIDDM Nephropathy Study Group," Diabetologia, Vol. 40, No. 7, 1997, pp. 816-823. doi: $10.1007 / \mathrm{s} 001250050754$

[4] W. M. McClellan, S. G. Satko, E. Gladstone, J. O. Krisher, A. S. Narva and B. I. Freedman, "Individuals with a Family History of ESRD Are a High-Risk Population for CKD: Implications for Targeted Surveillance and Intervention Activities," American Journal of Kidney Diseases, Vol. 53, Suppl. 3, 2009, pp. S100-S106.

[5] J. Coresh, E. Selvin, L. A. Stevens, J. Manzi, J. W. Kusek, P. Eggers, et al., "Prevalence of Chronic Kidney Disease in the United States," Journal of the American Medical Association, Vol. 298, No. 17, 2007, pp. 2038-2047. doi:10.1001/jama.298.17.2038

[6] Q. L. Zhang and D. Rothenbacher, "Prevalence of Chronic Kidney Disease in Population-Based Studies: Systematic Review," BMC Public Health, Vol. 8, 2008, pp. 117. doi:10.1186/1471-2458-8-117

[7] M. K. Haroun, B. G. Jaar, S. C. Hoffman, G. W. Comstock, M. J. Klag and J. Coresh, "Risk Factors for Chronic Kidney Disease: A Prospective Study of 23,534 Men and Women in Washington Country, Maryland," Journal of the American Society of Nephrology, Vol. 14, No. 11, 2003, pp. 2934-2941.

doi:10.1097/01.ASN.0000095249.99803.85

[8] F. C. Luft, "Hypertensive Nephrosclerosis: Update," Current Opinion in Nephrology and Hypertension, Vol. 13, No. 2, 2004, pp. 147-154. doi:10.1097/00041552-200403000-00002

[9] B. I. Freedman, S. S. Iskandar and R. G. Appel, "The Link between Hypertension and Nephrosclerosis," American Journal of Kidney Diseases, Vol. 25, No. 2, 1995, pp. 207-221. doi:10.1016/0272-6386(95)90001-2

[10] C. Y. Hsu, "Does Non-malignant Hypertension Cause Renal Insufficiency? Evidence-Based Perspective," Current Opinion in Nephrology and Hypertension, Vol. 11, No. 3, 
2002, pp. 267-272.

doi:10.1097/00041552-200205000-00001

[11] J. A. Sarnak, T. Greene, X. Wang, G. Beck, J. W. Kusek, A. J. Collins, et al., "The Effect of a Lower Target Blood Pressure on the Progression of Kidney Disease: LongTerm Follow-Up of the Modification of Diet in Renal Disease Study," Annals of Internal Medicine, Vol. 142, No. 5, 2005, pp. 342-351.

[12] A. Siewert-Delle, S. Ljungman, O. K. Andersson and L. Wilhelmsen, "Does Treated Primary Hypertension Lead to End-Stage Renal Disease? A 20-Year Follow-Up of the Primary Prevention Study in Göteborg, Sweden," Nephrology Dialysis Transplantation, Vol. 13, No. 12, 1998, pp. 3084-3090. doi:10.1093/ndt/13.12.3084

[13] C. Y. Hsu, C. E. McCulloch, J. Darbinian, A. S. Go and C. Iribarren, "Elevated Blood Pressure and Risk of EndStage Renal Disease in Subjects without Baseline Kidney Disease," Archives of Internal Medicine, Vol. 165, No. 8, 2005, pp. 923-928. doi:10.1001/archinte.165.8.923

[14] L. Zarif, A. Covic, S. Iyengar, A. R. Sehgal, J. R. Sedor and J. R. Schelling, "Inaccuracy of Clinical Phenotyping Parameters for Hypertensive Nephrosclerosis," Nephrology Dialysis Transplantation, Vol. 15, No. 11, 2000, pp. 18011807. doi:10.1093/ndt/15.11.1801

[15] R. B. Toto, "Hypertensive Nephrosclerosis in African Americans," Kidney International, Vol. 64, No. 6, 2003, pp. 2331-2341. doi:10.1046/j.1523-1755.2003.00333.x

[16] R. Marín, M. Gorostidi, F. Fernández-Vega and R. Álvarez-Navascués, "Systemic and Glomerular Hypertension and Progression of Chronic Renal Disease: The Dilemma of Nephrosclerosis," Kidney International Supplements, Vol. 99, Suppl. 99, 2005, pp. S52-S56. doi:10.1111/j.1523-1755.2005.09910.x

[17] W. M. McClellan, S. G. Satko, E. Gladstone, J. O. Krisher, A. S. Narva and B. I. Freedman, "Individual with a Family History of ESRD Are a High Risk Population for CKD: Implications for Targeted Surveillance and Intervention Activities," American Journal of Kidney Diseases, Vol. 53, Suppl. 3, 2009, pp. S100-S106. doi:10.1053/j.ajkd.2008.07.059

[18] W. H. Kao, M. J. Klag, L. A. Meoni, D. Reich, Y. Berthier-Schaad, M. Li, et al.,"MYH9 is Associated with Nondiabetic End Stage Renal Disease in African Americans," Nature Genetics, Vol. 40, No. 10, 2008, pp. 1185-1192. doi:10.1038/ng.232

[19] K. Anbazhagan, K. Sampathkumar, M. Ramakrishnan, S. Gomathi and G. S. Selvam, "Analysis of Polymorphism in Renin Angiotensin System and Other Related Genes in South Indian Chronic Kidney Disease Patients," Clinica Chimica Acta, Vol. 406, No. 1-2, 2009, pp. 108-112. doi:10.1016/j.cca.2009.06.003

[20] J. Xu , G. Li, P. Wang, H. Velazquez, X. Yao, Y. Li, et al., "Renalase Is a Novel, Soluble Monoamine Oxidase that Regulates Cardiac Function and Blood Pressure," Journal of Clinical Investigation, Vol. 115, No. 5, 2005, pp. 1275-1280.

[21] G. V. Desir, "Regulation of Blood Pressure and Cardiovascular Function by Renalase," Kidney International, Vol. 76, No. 2009, pp. 366-370. doi:10.1038/ki.2009.169

[22] G. Desir, P. Wang and H. Velazquez, "On the Mechani- sms Mediating the Cardioprotective Effect of Renalase," Journal of the American Society of Nephrology, 2009. $\mathrm{http}$ //www.asn-online.org/education_and_meetings/renal _week/archives/

[23] S. S. Ghosh, T. W. B. Gehr and D. A. Sica, "Renalase Regulates Blood Pressure in Salt Sensitive Dahl Rats," Journal of the American Society of Nephrology, Vol. 17, 2006, p. 208A.

[24] G. Desir, L. Tang, P. Wang, G. Li and H. Velazquez, “Antihypertensive Effect of Recombinant Renalase in Dahl Salt Sensitive (DSS) Rats," 2010.

http://www.asn-online.org/education_and_meetings/renal _week/archives/

[25] G. V. Desir, "Renalase Deficiency in Chronic Kidney Disease, and Its Contribution to Hypertension and Cardiovascular Disease," Current Opinion in Nephrology and Hypertension, Vol. 17, No. 2, 2008, pp. 181-185. doi:10.1097/MNH.0b013e3282f521ba

[26] M. Schlaich, F. Socratous and N. Eikelis, "Renalase Plasma Levels Are Associated with Systolic Blood Pressure in Patients with Resistant Hypertension," Journal of Hypertension, Vol. 28, 2010, pp. e434-e435. doi:10.1097/01.hjh.0000379519.82971.02

[27] Y. Wu, J. Xu, H. Velazquez, P. Wang, G. Li, D. Liu, et al., "Renalase Deficiency Aggravates Ischemic Myocardial Damage," Kidney International, Vol. 79, No. 8, 2011, pp. 853-860. doi:10.1038/ki.2010.488

[28] Q. Zhao, Z. Fan, J. He, S. Chen, H. Li, P. Zhang, et al., "Renalase Gene Is a Novel Susceptibility Gene for Essential Hypertension: A Two-Stage Association Study in Northern Han Chinese Population," Journal of Molecular Medicine, Vol. 85, No. 8, 2007, pp. 877-885. doi:10.1007/s00109-006-0151-4

[29] R. Farzaneh-Far, G. V. Desir, N. B. Schiller and M. A. Whooley, "A Functional Polymorphism in Renalase (Glu37Asp) Is Associated with Cardiac Hypertrophy, Dysfunction, and Ischemia: Data from the Heart and Soul Study," PLoS One, Vol. 5, No. 10, 2010, pp. e13496. doi:10.1371/journal.pone.0013496

[30] D. Ojea, R. Marin, E. Coto, et al., "Clinical and Genetic Basis of Hypertensive Nephrosclerosis," Nefrologia, Vol. 30, No. 6, 2010, pp. 687-697.

[31] A. S. Levey, J. Coresh, E. Balk, A. T. Kausz, A. Levin, M. W. Steffes, et al., "National Kidney Foundation Practice Guidelines for Chronic Kidney Disease: Evaluation, Classification, and Stratification," Annals of Internal Medicine, Vol. 139, No. 2, 2003, pp. 137-147.

[32] A. Fogo, J. A. Breyer, M. C. Smith, W. H. Cleveland, L. Agodoa, K. A. Kirk, et al., "Accuracy of the Diagnosis of Hypertensive Nephrosclerosis in African Americans: A Report from the African American Study of Kidney Disease (AASK) Trial. AASK Pilot Study Investigators," Kidney International, Vol. 51, No. 1, 1997, pp. 244-252.

[33] B. I. Freedman, P. J. Hicks, M. A. Bostrom, M. E. Cunningham, Y. Liu, J. Divers, et al., "Polymorphisms in the Non-muscle Myosin Heavy Chain 9 Gene (MYH9) Are Strongly Associated with End-stage Renal Disease Historically Attributed to Hypertension in African Americans," Kidney International, Vol. 75, No. 7, 2009, pp. 736-745. doi:10.1038/ki.2008.701 